\title{
Truck Essential Power Systems Efficiency Improvements for Medium-Duty Trucks
}

\author{
Final Report \\ Period Covered - 10/1/04 - 12/31/07 \\ Contractor: National Energy Technology Laboratory \\ Contract No.: DE-FC26-04NT42258
}

Principal Investigator/Technical Manager: Larry M. Slone

Caterpillar Inc. - Technology \& Solutions Division

14009 N. Old Galena Road

Mossville, IL 61552-7547

(309) 578-0243, fax: (309) 578-6285, e-mail: slone_larry_m@cat.com

Program Manager: Jeffrey F. Birkel

Caterpillar Inc. - Technology \& Solutions Division

(309) 636-1077, fax: (309) 636-2567, e-mail: birkel_jeffrey_f@cat.com

Consortium Team Members:

Dana Corporation

Engineered Machined Products (EMP)

Switched Reluctance Drives LTD (SRDL), division of Emerson Electric Co.

Project Manager: Carl Maronde

U.S. Department of Energy - National Energy Technology Laboratory

(412) 386-6402, fax: (412) 386-4775, e-mail: Carl.Maronde@netl.doe.gov

Technology Program Manager: Lee A. Slezak

U.S. Department of Energy - Office of the FreedomCAR and Vehicle Technology Program

(202) 586-2335, fax: (202) 586-2476, e-mail: lee.slezak@hq.doe.gov 


\section{DISCLAIMER}

This report was prepared as an account of work sponsored by an agency of the United States Government. Neither the United States Government nor any agency thereof, nor any of their employees, makes any warranty, express or implied, or assumes any legal liability or responsibility for the accuracy, completeness, or usefulness of any information, apparatus, product, or process disclosed, or represents that its use would not infringe privately owned rights. Reference herein to any specific commercial product, process, or service by trade name, trademark, manufacturer, or otherwise does not necessarily constitute or imply its endorsement, recommendation, or favoring by the United States Government or any agency thereof. The views and opinions of authors expressed herein do not necessarily state or reflect those of the United States Government or any agency thereof. 


\section{TABLE OF CONTENTS}

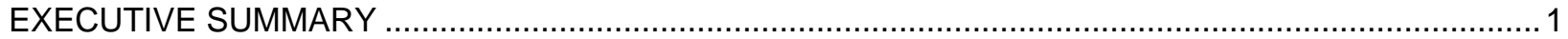

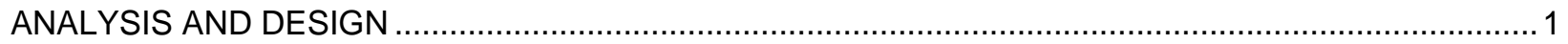

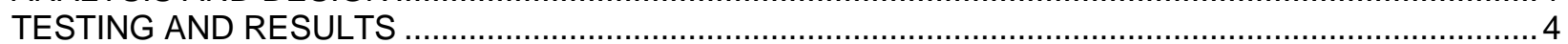

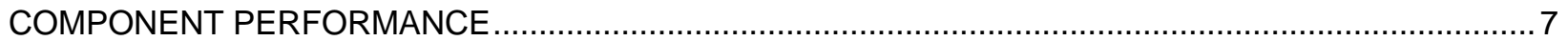

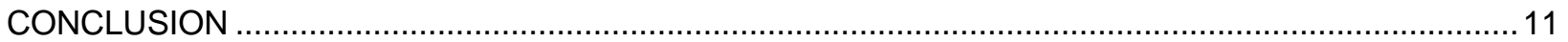

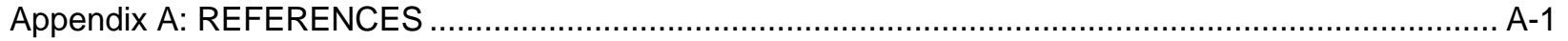

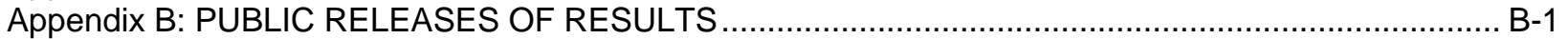

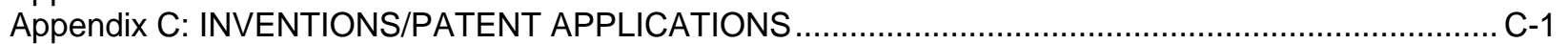




\section{EXECUTIVE SUMMARY}

With a variety of hybrid vehicles available in the passenger car market, electric technologies and components of that scale are becoming readily available. Commercial vehicle segments have lagged behind passenger car markets, leaving opportunities for component and system development. Escalating fuel prices impact all markets and provide motivation for OEMs, suppliers, customers, and end-users to seek new techniques and technologies to deliver reduced fuel consumption. The research presented here specifically targets the medium-duty (MD), Class 4-7, truck market with technologies aimed at reducing fuel consumption. These technologies could facilitate not only idle, but also parasitic load reductions.

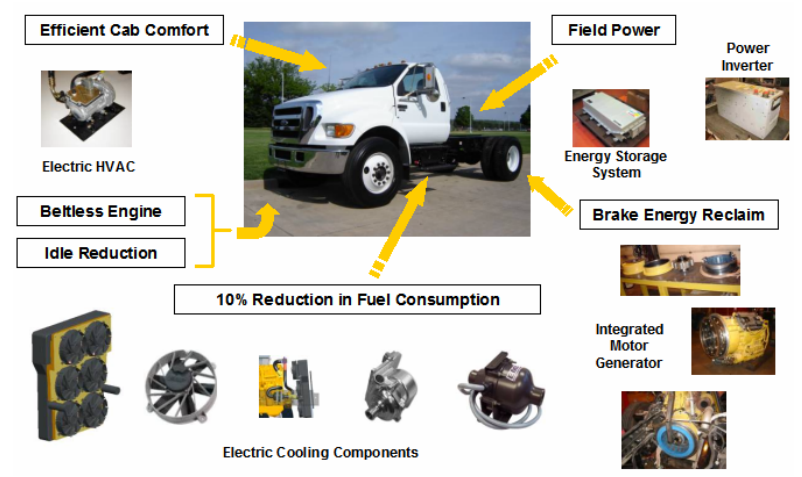

Figure 1 TEPS Testbed Truck

The development efforts here build upon the success of the More Electric Truck (MET) demonstration program at Caterpillar Inc. Employing a variety of electric accessories, the MET demonstrated the improvement seen with such technologies on a Class 8 truck. The Truck Essential Power Systems Efficiency Improvements for Medium-Duty Trucks (TEPS) team scaled the concepts and successes of MET to a MD chassis. The team designed an integrated starter/generator (ISG) package and energy storage system (ESS), explored ways to replace belt and gear-driven accessory systems, and developed supervisory control algorithms to direct the usage of the generated electricity and system behavior on the vehicle. All of these systems needed to fit within the footprint of a MD vehicle and be compatible with the existing conventional systems to the largest extent possible. The overall goal of this effort was to demonstrate a reduction in fuel consumption across the drive cycle, including during idle periods, through truck electrification. Furthermore, the team sought to evaluate the benefits of charging the energy storage system during vehicle braking.

The vehicle features an array of electric accessories facilitating on-demand, variable actuation. Removal of these accessories from the belt or geartrain of the engine yields efficiency improvements for the engine while freeing those accessories to perform at their individual peak efficiencies to meet instantaneous demand. The net result is a systems approach to fuel usage optimization. Unique control algorithms were specifically developed to capitalize on the flexibility afforded by the TEPS architecture. Moreover, the TEPS truck technology mixture exhibits a means to supplant current accessory power sources such as on-board or trailer-mounted gasoline-powered generators or air compressors. Such functionality further enhances the value of the electric systems beyond the fuel savings alone.

To demonstrate the fuel economy improvement wrought via the TEPS components, vehicle fuel economy testing was performed on the nearly stock (baseline) truck and the TEPS truck. Table 1 illustrates the fuel economy gains produced by the TEPS truck electrification.

Table 1 Fuel Economy Test Results

\begin{tabular}{|l|c|}
\hline & Average Fuel Economy (mpg) \\
\hline Baseline & 7.82 \\
\hline TEPS & 9.04 \\
\hline
\end{tabular}

While the fuel economy results shown in Table 1 do reflect specific test conditions, they show that electrification of accessory hardware can yield significant fuel savings. In this case, the savings equated to a 15 percent reduction in fuel consumption during controlled on-road testing. Truck electrification allows engine shutdown during idle conditions as well as independent ondemand actuation of accessory systems. In some cases, independent actuation may even include lack of operation, a feature not always present in mechanically driven components. This combination of attributes allows significant improvements in system efficiency and the fuel economy improvements demonstrated by the TEPS team.

\section{ANALYSIS AND DESIGN}

Medium-duty trucks present a unique challenge in the breadth of vocations they serve and the multitude of manufacturers and outfitters present. In contrast to most passenger car and Class 8 vehicles that largely travel from the manufacturer to a dealer lot for purchase by an end-user, 
medium-duty trucks typically leave the factory bound for a body builder. These chassis leave the original equipment manufacturer (OEM) as a drivable cab and rails arrangement, without any sort of bed or box on the frame rails behind the cab. Body building companies then outfit a truck with appropriate hardware for a specific vocation or application.

Body builders convert the simple cab and rails vehicles into the tow trucks, ambulances, beverage trucks, etc. more commonly seen on roadways. As such, the TEPS design effort has focused on delivering components and systems that can be applied without encroaching on the packaging envelope of a conventional truck. Additional value for the TEPS components may be demonstrated via assorted power sources enabled by the electrified architecture.

\section{Cooling Module}

The cooling module defined for this application includes the pumps, fans, and heat exchangers employed by both the powertrain and TEPSspecific electrical systems. The cooling module represents an ideal location to utilize the availability of electricity for driving accessories. Conventionally, cooling module components are primarily driven mechanically from the engine. These mechanical connections are prime candidates for independent, on-demand operation rather than operating points dictated by engine speed. Independent operation improves the efficiency of the entire system and removes unnecessary parasitic loads from the engine.

The TEPS truck contains two coolant circuits, one high temperature and one low temperature loop. The major constituents of the high temperature loop are the engine, transmission, and ISG stator. The heat load of this circuit is approximately 150 $\mathrm{kW}$. The low temperature loop, on the other hand, cools the power electronics for all of the TEPS devices as well as the ESS. The heat load of this circuit is approximately $20 \mathrm{~kW}$.

Most of the TEPS cooling module components operate directly from the 340 volt direct current (VDC) produced by the ISG to yield the best system efficiency. For the purposes of this effort, 340 volts is considered high voltage (HV) while low voltage (LV) nomenclature identifies the conventional 12 -volt system. The engine jacket water pump provides a prime example of the flexibility an electrified architecture offers while running efficiently on 340 VDC. The HV pump circulates coolant through the high temperature circuit. Meanwhile, an array of HV electric fans replaced the traditional, single engine-driven fan as well. The fan array offered the TEPS team opportunities to optimize fan operation around the specific needs of individual truck systems. Low voltage cooling components include a coolant pump and valve. A LV pump is employed to circulate coolant to the low temperature coolant circuit. Finally, an electronically-controlled LV valve replaces the conventional wax-type thermostat. These components provide a system with fine levels of control and improved efficiency.

\section{Energy Storage System}

Harnessing the output power of the ISG is the energy storage system. Much of the early analytical work focused on determining the appropriate ESS specifications for the TEPS system. Toward this end, the TEPS team developed a dynamic plant model for the purposes of evaluating and selecting the ESS. Numerous drive cycles, applications, chemistries, and storage sizes were considered in the analysis. Key matters for the selection were idle reduction capability, durability, commercial availability, and life expectancy. An important consideration for ESS life is the depth of discharge (DOD) utilized by the control algorithms. Research conducted by Caterpillar estimates the expected ESS life cycles based on DOD.

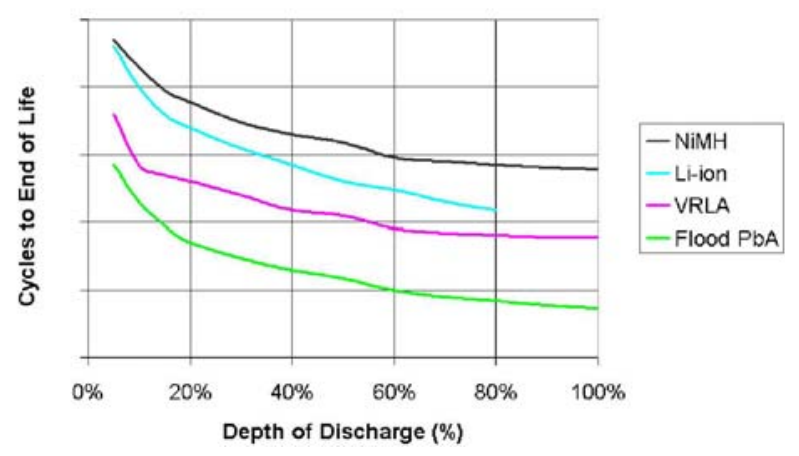

Figure 2 Life Estimation Plot for ESS

Simulations determined the requirements to achieve a minimum four-year life while meeting the cycle requirements. The four ESS chemistries of Figure 2 were each run against representative medium truck cycles. Aside from the life cycle requirements, idle reduction capability at a job site also influenced ESS sizing. In one example, for instance, this required the ability to operate at the simulated job site for a period of at least 20 minutes prior to restarting the engine to replenish the batteries. Wherever possible, the assumed "battery pack" sizes roughly represented systems 
or cells which were commercially available at the time.

This application required a significant capacity for DOD and life, which in some cases necessitated an exceedingly large pack size. Even though the valve regulated lead acid (VRLA) battery had superior power and life expectancies, the weight of the VRLA battery represented a significant hurdle. While lithium-ion (Li-lon) offered a lighter package with more power for equivalent life, the commercial maturity and availability of packaged solutions led the team to select nickel metal hydride (NiMH) batteries. Per Table 2, a $1.5 \mathrm{kWh}$ pack met the application requirements. The team, however, implemented a readily available, albeit slightly oversized, $2.4 \mathrm{kWh}$ pack.

\section{Table 2 Battery Simulation Results Comparison}

\begin{tabular}{|l|c|c|c|}
\hline $\begin{array}{l}\text { Battery } \\
\text { Technology }\end{array}$ & $\begin{array}{c}\text { Size } \\
\text { (Wh) }\end{array}$ & $\begin{array}{c}\text { Mass } \\
\text { (kg) }\end{array}$ & $\begin{array}{c}\text { Life } \\
\text { (years) }\end{array}$ \\
\hline VRLA & 16000 & 400 & 5 \\
\hline NiMH & 1500 & 46 & 4 \\
\hline Li-Ion & 2000 & 33 & 4 \\
\hline
\end{tabular}

\section{Integrated Starter/Generator}

The heart of the electrification effort is the integrated starter/generator, a switched reluctance machine packaged in the flywheel housing of a Caterpillar C7 engine. The ISG starts the engine, serves as the lone electrical generator, and provides motive power to the driveline. This machine operates at approximately 90 percent efficiency across the operating range of the engine.

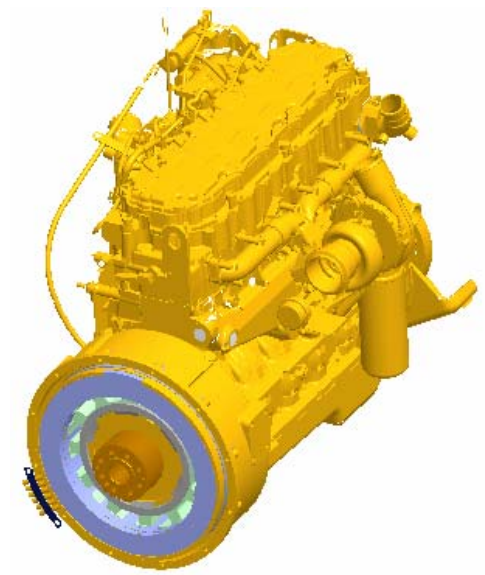

\section{Figure 3 Caterpillar ${ }^{\circledR}$ C7 Engine with ISG}

Compared to conventional alternators which operate at 45-55 percent efficiency ${ }^{2}$, the ISG represents a substantial improvement in power generation efficiency. The ISG is rated for $20 \mathrm{~kW}$ of generating power above 1300 RPM. Conversely, the machine is capable of delivering $12 \mathrm{~kW}$ of motoring power across most engine operating speeds.

The design of the ISG is based on the proven design found in the MET. ${ }^{2}$ Like the previous design, the TEPS ISG operates around a nominal bus voltage of 340 VDC when charging. The stator can be suitably cooled with jacket water from the engine up to temperatures of $100{ }^{\circ} \mathrm{C}$. Nevertheless, this new design represents a refinement of the earlier design - incorporating lessons learned from earlier projects. In particular, cable routings have been altered to facilitate assembly on an engine while sensor-less position detection algorithms eliminate the need for rotorposition sensors. The machine has been designed to deliver not only engine starting power, but also a useful power curve for providing some additional propulsive power.

This latest ISG power converter unit benefits from improvements to both the controller board and power conversion hardware brought about by several years of technological advancements. The current iteration represents a near 40 percent reduction in size compared to the controller produced previously.

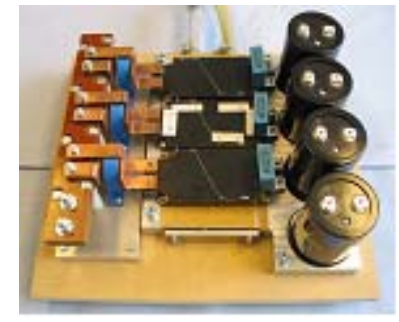

Figure 4 ISG Power Converter

\section{Demonstration Platform Selection}

The project team used extensive simulation and analysis in the conceptualization and component design for the truck. In order to design the TEPS components to be readily applicable to a breadth of medium-duty applications, the team considered a variety of vocations.

For example, a general pickup and delivery cycle was selected to embody a variety of medium-duty applications. The cycle features a top speed of approximately $49 \mathrm{mph}$ and an average speed of about $19 \mathrm{mph}$, as well as some brief idling periods. Figure 5 highlights the results of the pickup and delivery cycle simulations. The simulations suggested fuel consumption reductions of more than 10 percent on this cycle. 


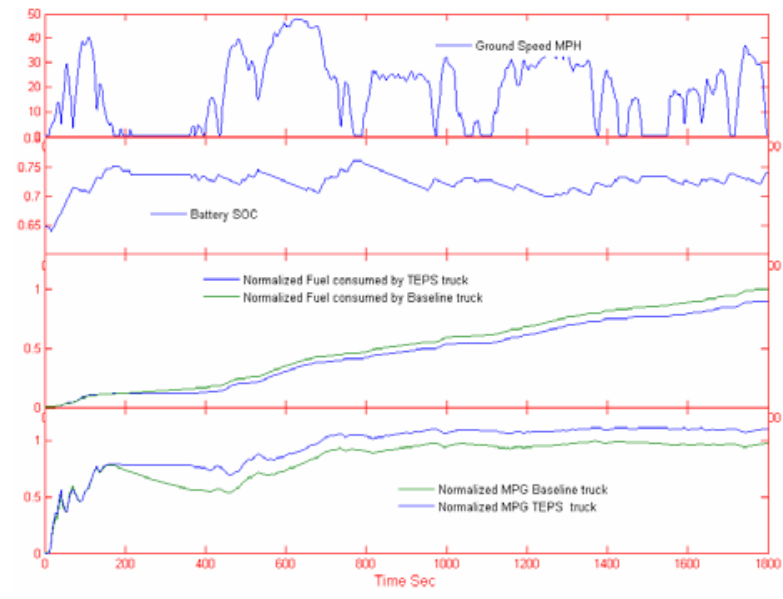

Figure 5 Pickup and Delivery Simulation Results

After careful analysis of several medium-duty applications, the TEPS team decided to build the demonstration platform as a utility truck. Utility trucks operate under cycles that may involve extended idling periods as well as a need for field power. Both of these requirements are easily facilitated by an electrified architecture implemented with energy storage. Thus, the utility truck represented an ideal platform for showcasing the advanced technologies of the TEPS project.

In the absence of "standard" utility truck operating cycles, a cycle was constructed to simulate utility truck activities. The cycle consisted of road driving speeds up to approximately $38 \mathrm{mph}$, stops at traffic lights, and a job site idle period of about 90 minutes. Accessory loads simulating the use of a bucket and/or tool were included in the plant model. The simulation results for this profile (as shown in Figure 6) yielded fuel consumption reductions of approximately 40 percent, with savings during the idle periods topping 50 percent.

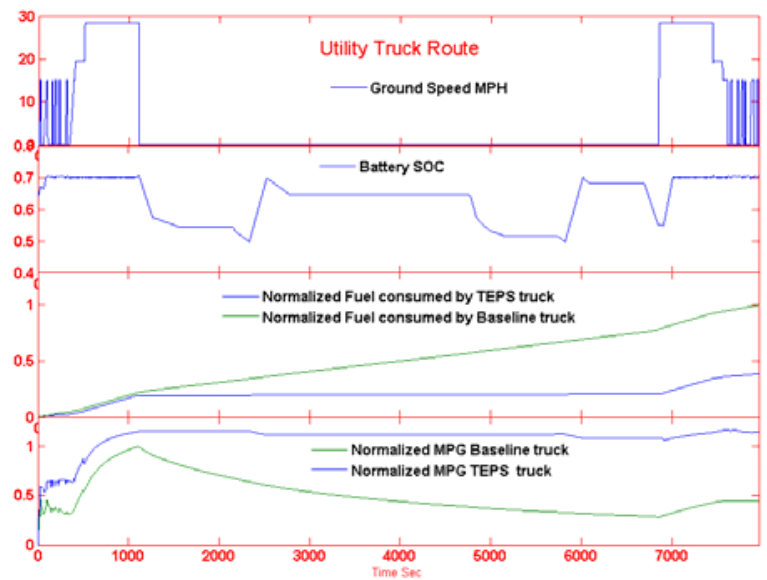

Figure 6 Utility Route Simulation Results
Simulations of the intended fuel economy test route were also conducted. The route stems from an established test procedure adapted to the test facility. The route is a geometric trace of accelerations and decelerations designed to mimic typical driving conditions. All acceleration events are performed at full throttle. Idle time appended to the end of the run emulates job site activity. The simulations of this route indicated potential fuel savings on the order of $15 \%$.

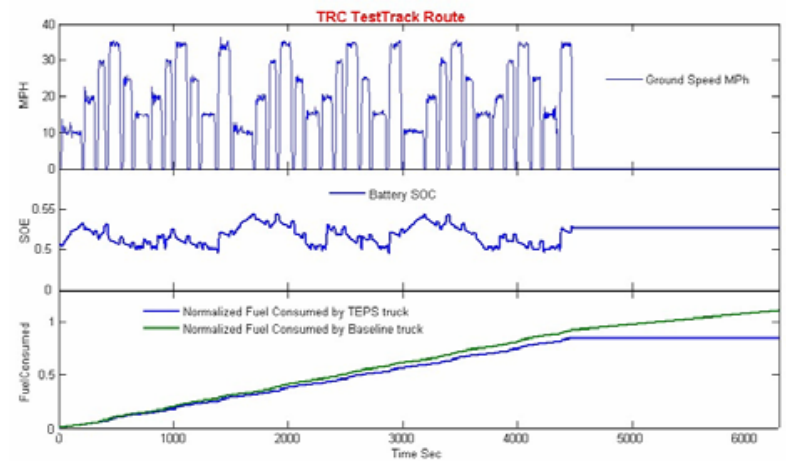

Figure 7 Test Track Simulation Results

\section{TESTING AND RESULTS}

Caterpillar contracted an independent proving ground, the Transportation Research Center (TRC), to perform vehicle testing. The 4500-acre site of TRC comprises facilities for both on-road and dynamometer testing. A truck similar to the initial specifications for the TEPS test truck was acquired to serve as a control vehicle for testing. The control truck served as a reference point for fuel economy comparisons at each stage of the test truck's lifecycle and to account for variability in the test environment. Both trucks were ballasted to $19,210 \mathrm{lbs}$ for the duration of testing to simulate an average load for a Class 6 vehicle. When appropriate, vehicles were driven on the test track for approximately 30 minutes prior to testing to bring all systems up to operating temperatures.

\section{Coastdown Testing}

TRC performed coastdown testing in accordance with Society of Automotive Engineers (SAE) J1263 protocol. The test determines the forces required of the vehicle to maintain a steady state speed on a level roadway under no wind conditions. This test is also used to determine the coefficient of aerodynamic drag (CD) of the truck. The coastdown testing data was utilized for plant model validation as well as chassis dynamometer parameter information. Testing was conducted in the evening when weather forecasts indicated wind speed would be minimal (not in excess of 
approximately $2 \mathrm{mph}$ ). The test truck was then driven into a two-mile long straight area of the track at a speed of $65 \mathrm{mph}$ and shifted into neutral and allowed to coast. One run was done moving in a northbound direction and one was completed in a southbound direction. The procedure was repeated until the team had 7 run pairs completed. Upon completion, the vehicle with the driver was weighed and the post-test weight used for data analysis.

\section{Table 3 Medium-Duty Truck Coastdown Results Summary}

\begin{tabular}{|l|l|}
\hline A0 Coefficient & 0.1587743 \\
\hline A2 Coefficient & $0.1282783 \mathrm{E}-03$ \\
\hline CD & 0.772 \\
\hline Hp Req'd @ 50 $\mathbf{~ m p h}$ & 557.7 \\
\hline Hp Req'd @ 60 mph & 866.3 \\
\hline Hp Req'd @ 70 $\mathbf{~ m p h}$ & 1282.2 \\
\hline Hp Req'd @ 80 $\mathbf{~ p h}$ & 1823.5 \\
\hline
\end{tabular}

The CD indicates the relative amount of aerodynamic drag the vehicle produces. A lower $C D$ value indicates greater efficiency. As seen in Table 3, the baseline truck, without TEPS components, produced a CD of 0.772 . Finally, TRC measured the frontal area of the truck to be $8324 \mathrm{in}^{2}$. Frontal area and CD values were not expected to change significantly after the TEPS integration effort. Therefore, the team considers the initial values valid for the TEPS truck and elected to not perform a second evaluation of these traits.

\section{Acceleration Testing}

TRC executed acceleration testing in compliance with SAE J1491 to determine the time required to reach $60 \mathrm{mph}$ from a stop. This data was then used to calculate the maximum road grade the vehicle can climb at $40 \mathrm{mph}$. One run in the northbound direction and one in the southbound comprise a run pair. Run pairs were performed until three sets of measurements were completed within the acceptable testing limits. Compromised runs and their paired run were excluded and another pair of runs completed. Test results represent the average of three pairs that met the variability requirements of SAE J1491. By curve fitting the acceleration data, TRC determined the maximum road grade that the vehicle can climb at $40 \mathrm{mph}$ to be 5.5 percent on the southbound route and 5.9 percent on the northbound route.

Baseline testing revealed an average time of 36.62 seconds for the vehicle to reach $60 \mathrm{mph}$. Time and weather constraints, however, prohibited like testing of the TEPS truck. Nevertheless, an acceleration comparison between the TEPS and baseline configurations was possible using test cycle data. During the drive cycles, the trucks were accelerated at wide-open throttle. The top speed during the cycle was approximately $35 \mathrm{mph}$, therefore for both the baseline and TEPS configurations, multiple data points were available to estimate the $0-35 \mathrm{mph}$ acceleration performance. Table 4 shows the acceleration results for the two configurations. Multiple points were taken; therefore, variation in the data has been included. The data shows that the acceleration for the TEPS truck is comparable to the baseline truck within the experimental variation.

Table 4 Baseline and TEPS Acceleration

\begin{tabular}{|l|c|}
\hline & $\mathbf{0 - 3 5} \mathbf{~ m p h}$ Acceleration (sec) \\
\hline Baseline & $13.75 \pm 0.51$ \\
\hline TEPS & $14.03 \pm 0.41$ \\
\hline
\end{tabular}

\section{Steady State Dynamometer Cooling Testing}

Steady state dynamometer testing established a baseline for the performance of the standard cooling package across a range of loads, engine speeds, and ambient temperatures. The truck was equipped with fuel flow meters and thermocouples in its cooling heat exchangers to gather data about fuel usage and heat rejection. The TEPS team intended to compare cooling performance and steady state fuel consumption between the baseline and the TEPS truck. Unfortunately, a component failure prohibited the full battery of tests on the TEPS truck, though sufficient data was collected to suggest that the TEPS cooling system might nearly match the performance of the baseline system. Furthermore, successful on-road fuel economy testing provided more than adequate data for detailing the efficiency improvements of the TEPS systems.

\section{Fuel Economy Testing}

Fuel economy testing was performed per the standards set forth in the SAE J1321 test procedure. A test run featured 6 laps of a 7.5-mile track for a total distance of 45 miles. The tests were broken up into sets of 3 laps with idle time in the middle to emulate field activity. Each driver remained paired with the same truck and the drivers tested the trucks at speeds ranging from $10 \mathrm{mph}$ to $35 \mathrm{mph}$, simulating a nominal pickup and delivery cycle. Gravimetric tanks enabled direct measurement of the fuel mass consumed during test runs. Both the test and control truck were tested simultaneously on the track, with one 
truck embarking on the route two minutes ahead of the other. The test and control trucks ran through a minimum of 3 runs for each test segment. In order for the run sets to be considered successful, the fuel consumption test to control ( $\mathrm{T} / \mathrm{C})$ ratio of each run needed to be within 2 percent of the other runs in that set. Also, over the course of the 3 runs, the run times needed to be within +/- 0.5 percent of the other runs in that set. Run data that was not within those parameters was disregarded and the run was repeated.

After the installation of a Caterpillar CX28 transmission into the TEPS test truck, an initial fuel economy test was conducted to provide a baseline for comparison to the TEPS electrified truck. The fuel consumption and component data for this round of testing served as the basis for comparison to the electrified chassis testing. As indicated in Figure 8, the accessory loads on the baseline truck utilized approximately 15 percent of the fuel consumed during the entire test run.

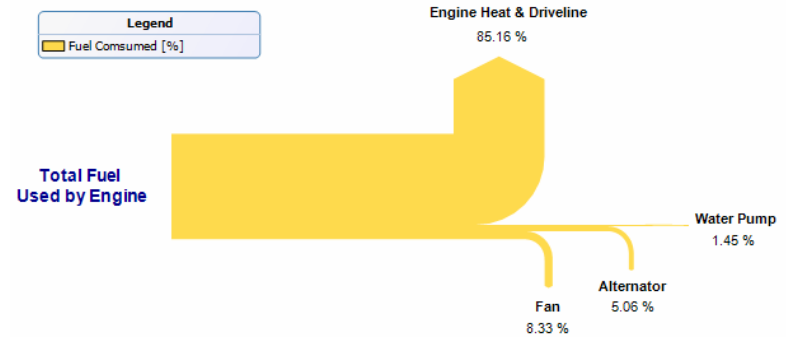

Figure 8 Baseline Fuel Distribution

After integration of the TEPS components, the test truck returned to TRC and was again subjected to fuel economy testing alongside the same control truck. The testing procedure and route were replicated from the baseline testing. The overall results of this testing show that the baseline Ford F-750 gave an average T/C ratio of 1.1800 with an average fuel economy of $7.82 \mathrm{mpg}$. The TEPS truck gave an average T/C ratio of 1.0023 with an average fuel economy of $9.04 \mathrm{mpg}$.

Table 5 Fuel Economy Test Results

\begin{tabular}{|l|c|c|}
\hline & $\begin{array}{c}\text { Average T/C } \\
\text { Ratio }\end{array}$ & $\begin{array}{c}\text { Average Fuel Economy } \\
\text { (mpg) }\end{array}$ \\
\hline Baseline & 1.1800 & 7.82 \\
\hline TEPS & 1.0023 & 9.04 \\
\hline
\end{tabular}

The results of Table 5 reveal the 15.1 percent reduction in fuel consumption for the TEPS truck relative to the baseline non-electrified truck. Figure 9 shows the normalized fuel consumption for the two different trucks over the test cycle. The plot also breaks down the fuel savings as a function of the driving portion of the cycle versus the idling portion of the cycle.

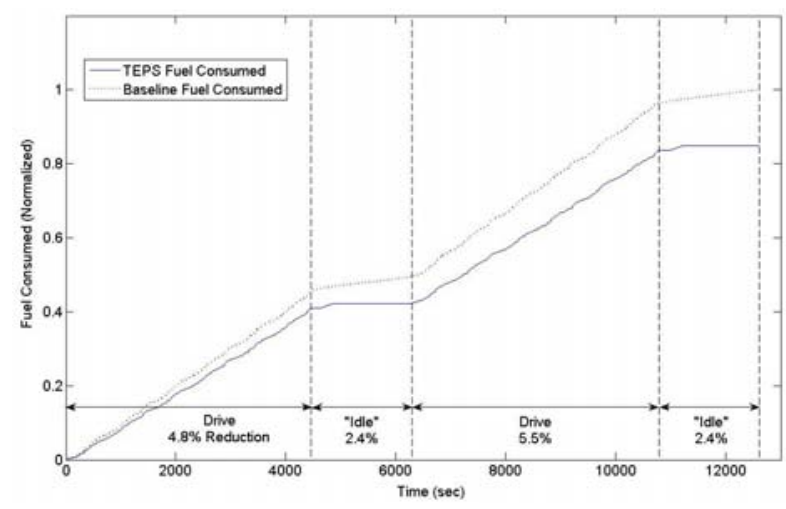

Figure 9 Fuel Savings by Cycle Portion

The data shows that 10.3 percent of the fuel savings occurs during the driving portion of the cycle while 4.8 percent occurs during the idling portion of the cycle. While there is a difference in fuel savings between the two driving portions of the cycle, this difference is within the acceptable range set by the test procedure in terms of fuel volume measurement between cycles. Due to the nature of the idling portion of the cycle, it was determined that isolating the driving portion of the cycle might prove useful for analysis purposes. The fuel consumption of the TEPS truck during the idling portion of the cycle is heavily based on the idle duration. Engine cycling during this period results in rate or time-based savings.

Table 6 Fuel Consumption and Economy Improvements

\begin{tabular}{|l|c|}
\hline \multicolumn{2}{|c|}{$\begin{array}{c}\text { Fuel Consumption Improvement } \\
\text { (Percent of Mass of Fuel Consumed) }\end{array}$} \\
\hline Driving & $10.3 \%$ \\
\hline Idling & $4.8 \%$ \\
\hline Overall & $15.1 \%$ \\
\hline \multicolumn{2}{|c|}{ Fuel Economy Improvement } \\
(Percent of Mass of Fuel Consumed per Given Distance) \\
\hline Driving & $11.5 \%$ \\
\hline
\end{tabular}

As noted in Table 6, the 10.3 percent fuel savings for the driving portion of the cycle translates into an 11.5 percent improvement in fuel economy. Figure 10, meanwhile, shows the drive cycle along with the fuel consumed and the ESS state of charge (SOC). 


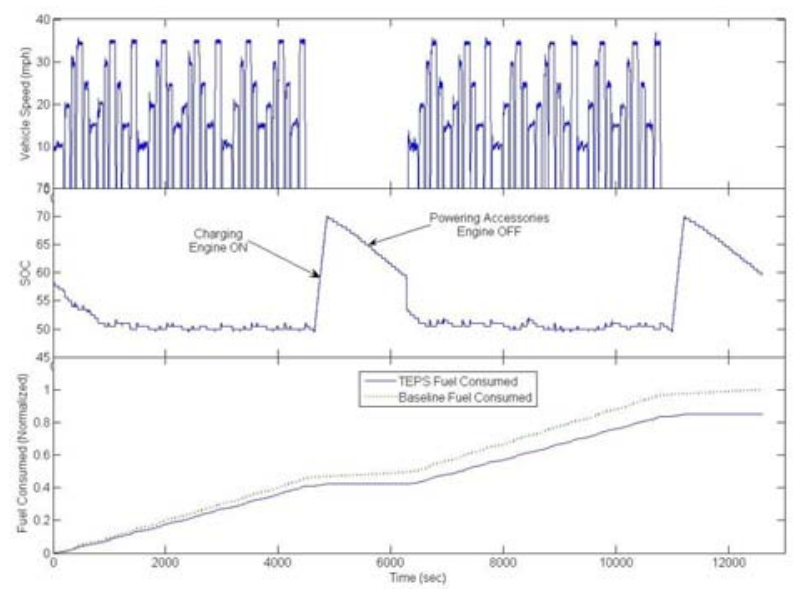

Figure 10 TRC Test Results for SOC and Fuel

The SOC is maintained at a level near the minimum with the constant starts and stops of the driving portion of the cycle. When the idling portion begins and the engine shuts off, the SOC begins to drop as the battery powers the accessories until it is at the minimum allowed value. Once the SOC reaches that minimum allowed value, the engine turns back on and charges the batteries to the maximum allowable SOC. With the batteries recharged, they can now power the accessory load through the end of the idle portion with the engine off. At the time the predetermined idle period has ended and the next drive portion begins, the SOC has not reached the minimum value. The remaining charge implies that the batteries could have powered the accessories for a longer period of time with the engine off. This phenomenon reinforces the notion of time-based savings and suggests that additional fuel savings would continue to accrue if the truck had continued the idle period.

This charging and discharging of the batteries is highly dependent of the accessory load. In Figure 10 , the accessory load during the idling period was approximately $0.75 \mathrm{~kW}$ and the batteries were charged at approximately $10 \mathrm{~kW}$. The system offers the flexibility to elevate idle speed to maintain or improve fuel efficiency under various loading conditions. In applications such as a service truck, there might be increased accessory loads causing a higher frequency of battery recharging. Even with this increased engine-on time, there would still be fuel savings over the baseline configuration that would be subject to the same accessory loads.

The distribution of fuel usage in the TEPS truck is shown in Figure 11. In comparison to the baseline testing data, the TEPS truck consumes approximately 6.5 percent of the fuel to power accessories. While there are more than twice as many accessories, their efficient operation means that they still consume less than half of the relative fuel that the mechanical accessories required. Given that the powertrain will require the same power and energy to meet the driving cycle, the figure provides an additional means of considering the fuel savings netted from the TEPS configuration.

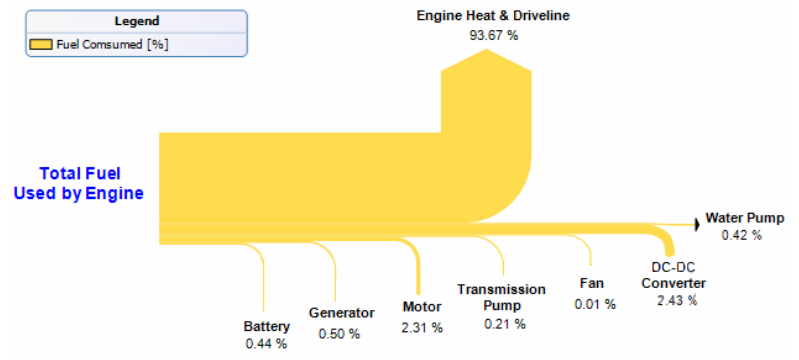

Figure 11 TEPS Fuel Distribution

Note that Figure 11 does not reflect the impact of brake energy reclamation, merely accounting for the expenditure of fuel. Brake energy reclamation would only serve to make the generator appear more efficient since more energy is produced from the same quantity of fuel. The Component Performance section further outlines the contributions of the TEPS components.

\section{COMPONENT PERFORMANCE}

Through the course of the baseline and TEPS testing, various component performance parameters were measured for the purpose of evaluating the relative performance of the baseline and TEPS components. The purpose of evaluating the component performance was to determine the contributions of specific components to the overall fuel savings. Such knowledge could facilitate improvements in overall system efficiency by focusing efforts on the components offering the greatest benefit.

Equation 1 depicts the formula used to approximate the fuel consumed by each component. When computing fuel consumption for each accessory, losses in the generator and battery were considered separately. 


$$
m_{\text {fuel }}=\int_{o}^{T} \frac{P_{\text {ICE }}}{\eta_{\text {ICE }}\left(Q_{\text {fuel }}\right)} \cdot d T
$$

where $m=$ Fuel Mass

$$
\begin{aligned}
& \mathrm{P}=\text { Engine Power } \\
& \eta=\text { Engine Efficiency } \\
& \mathrm{Q}=\text { Fuel Specific Energy }
\end{aligned}
$$

\section{Equation 1 Fuel Consumption Calculation}

The computed fuel consumption values were compared to the computed totals from the sensor data on the vehicle in order to validate the computations. Fuel totals compared to within 1 percent. The total fuel usage rate computed from sensor data on the vehicle was then compared to the fuel mass data collected during the road testing. These totals compared with a difference of 3 percent. The consistency of the results reinforced the value of the methods applied.

As suggested, the component fuel consumption can highlight areas of significant opportunity for efficiency enhancements. Figure 12 displays the respective fuel consumption for the accessories.

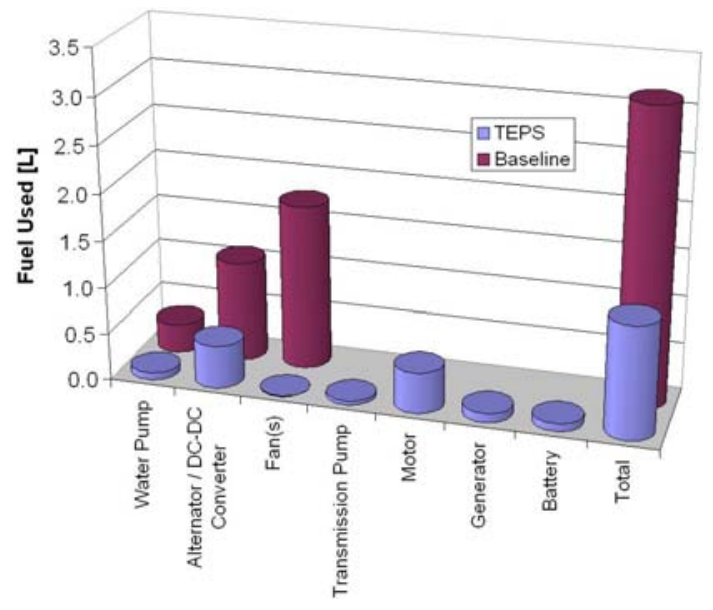

Figure 12 Component Fuel Consumption

Again, while the numerical count of accessories has more than doubled, the fuel consumption of the lot has been cut by more than half. In fact, a mere $1.07 \mathrm{~L}$ of fuel powered the TEPS accessories in contrast to the $3.13 \mathrm{~L}$ burned during baseline testing. These figures reinforce the value of component design and operation that is not tailored solely to a peak power or peak torque condition. Subsequent sections of this report provide additional details on the specific analysis of each component.

\section{Cooling System Fan}

The TEPS team assessed the potential benefits of replacing a belt-driven cooling system fan with a parallel array of variable-speed, electrically driven fans. Removal of the viscous fan clutch achieved significant savings. During baseline testing, the viscous fan clutch never completely disengaged. Internal friction of the clutch drove the fan at a minimal speed even when the fan was not required for cooling. This fan behavior creates a speed-varying parasitic loss. The use of electric fans in the TEPS system eliminated this parasitic loss completely by operating the fans only when necessary. The use of an array of electrically powered fans permitted intelligent control of the fans and localization of specific fans to specific tasks, increasing system flexibility. Figure 13 shows the arrangement of the fans relative to their respective heat exchangers. Two fans, for instance, cool the air-to-air aftercooler (ATAAC) while the remaining four cool the radiator. Finally, the bottom two also cool the low temperature radiator, thus localizing cooling to the required component(s).

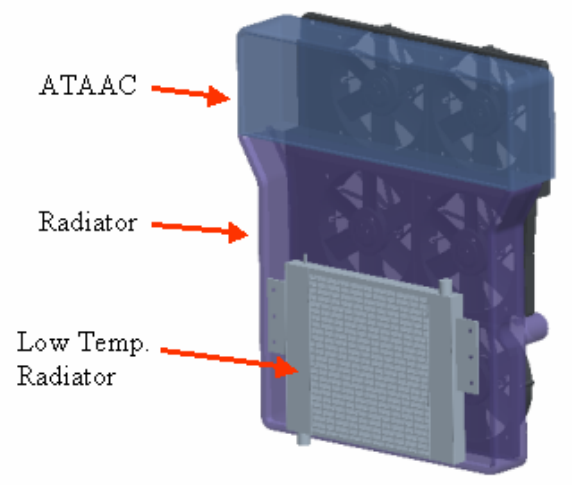

Figure 13 TEPS Cooling Module

The TEPS cooling system utilized an electric thermostat valve controlled in a manner to achieve a set-point temperature of $98{ }^{\circ} \mathrm{C}$. Having the flexibility to control the fans electrically allowed the option of directing all of the coolant flow through the radiator with the electric thermostat valve before activating the fans to further minimize fan operation.

The performance measures were quantified for the baseline version of the truck by installing a proximity detector on the front of the engine to measure the speed. The OEM fan turned throughout the baseline testing. The torque-speed characteristic of the fan was then measured and applied to the speed measured during testing to determine the power draw of the fan during the 
course of the test. Through the course of the baseline test, the mechanical fan ran at a speed related to the engine speed by the dynamic friction of the viscous clutch. This resulted in an average power draw of $1.8 \mathrm{~kW}$ despite engine coolant temperature never exceeding $89^{\circ} \mathrm{C}$.

The power draw of the TEPS configuration was determined by measuring the power extracted by the specific fan inverters using a 1 percent 0.05 ohm series resistor and measuring the voltage rise across the resistor with an analog-to-digital (A/D) converter. This value was multiplied by an A/D bus voltage reading to obtain the power value. The TEPS configuration only required operation of two of the fans for a very short duration to cool the ATAAC.

Baseline and final testing were performed in autumn, under seasonably cool temperatures. The cooler ambient temperatures undoubtedly contributed to lower engine temperatures minimizing the need for cooling fan operation. Nevertheless, significant fuel consumption reductions would still be expected in more challenging conditions. In fact, the independent actuation of the fans can lead to steady state operation that represents a vast improvement over the cyclic operation of a conventional mechanical fan. ${ }^{4}$

In this case, the mechanical fan utilized 8.3 percent of the fuel during baseline testing. The electric fans used on the TEPS truck consumed only about 0.02 percent of the fuel. Electrification of the cooling fans reduced their fuel consumption by 99.8 percent. Although the road test weather fell short of peak load conditions, the independent, variable speed nature of the TEPS accessories should provide substantial savings throughout their operating envelope.

\section{Engine Coolant Pump}

The engine coolant pump was perceived as another possible opportunity for fuel savings. The baseline, non-electrified configuration employs a pump with a belted connection to the engine crankshaft. Pump electrification allows the supervisory controller to vary pump speed according to the flow required for the specific operating condition. In contrast, mechanical pumps provide enough flow for the maximum cooling requirement at a given speed. The resultant pump size may yield inefficient operation during lower loading conditions. Moreover, Figure 14 emphasizes the flexibility in mounting afforded by electric accessories. In the TEPS truck, the electric coolant pump is mounted inside the frame rail, isolating it from direct engine heat and vibration.

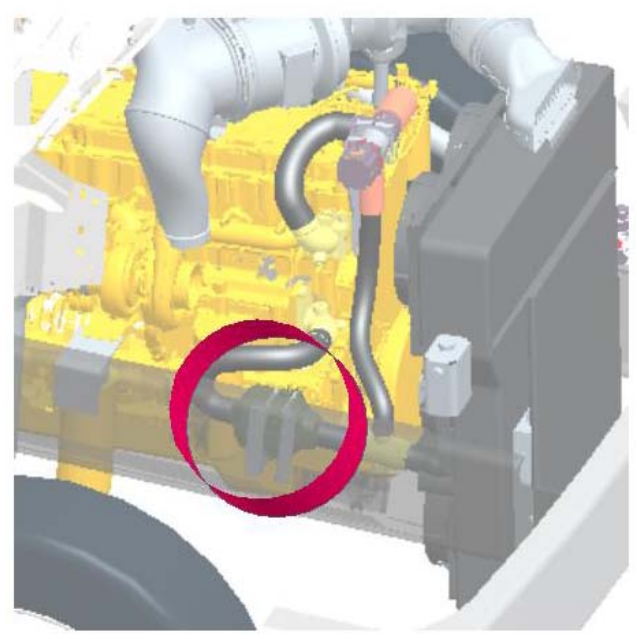

Figure 14 Electric Coolant Pump

The supervisory controller utilized an algorithm to regulate the cooling temperature to $98^{\circ} \mathrm{C}$ using the electric valve, the cooling fans, and the coolant pump. A primary role of the coolant pump is to limit the temperature rise across the engine to a safe level. As such, the coolant pump control focused primarily on maintaining a coolant temperature rise across the engine block of $3{ }^{\circ} \mathrm{C}$. In the event the coolant temperature would rise close to the maximum allowable temperature, the control provided functionality to run the coolant pump at maximum speed to reduce the coolant temperature rise throughout the circuit, thereby slightly reducing the maximum temperature.

During baseline testing, the mechanical coolant pump speed was tied directly to engine speed. Utilizing the characteristic curve of Figure 15 and the speed data logged during testing, pump power was calculated throughout the cycle. During TEPS testing, the electric coolant pump inverter measured the pump speed. Similar to the mechanical unit, the electric pump was characterized based upon the electrical power draw versus speed in the pumping circuit. Again, application of this characteristic curve to measured pump speed provides a power value to use when calculating the fuel consumption of the component. As seen in Figure 15, the polynomial equations developed fit the test data quite well, facilitating the power analysis. 


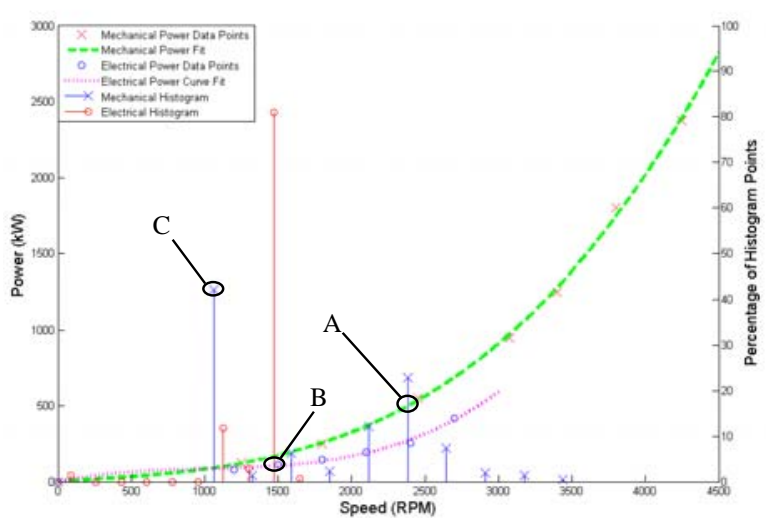

Figure 15 Coolant Pump Characteristics

A simple analysis of the energy consumption can be established by considering three primary data points in Figure 15. As the histogram data alludes, point ' $A$ ' represents a typical operating point for the mechanical coolant pump for the driving portion of the cycle. Point ' $\mathrm{B}$ ', meanwhile, indicates the same for the electric cooling pump. From these points, it can be seen that the electric pump will average slightly less power draw than the mechanical pump. Initially, the difference might seem small. Nevertheless, the difference amounts to nearly $200 \mathrm{~W}$ less draw at any moment throughout the cycle.

Via the histogram data, point ' $C$ ' shows the idle portion operating point for the mechanical pump. Note that the power consumed by the mechanical pump at point ' $C$ ' is nearly equivalent to the steady state operating point ' $A$ ' for the electric pump. In practice, however, the electric water pump can be powered down during engine-off periods to further reduce energy use.

During baseline testing, the OEM mechanical coolant pump was responsible for 1.45 percent of the fuel consumed. The electric coolant pump used on the TEPS truck was responsible for 0.42 percent of the fuel consumed during testing. Electrification of the coolant pump reduced fuel consumption by 73.9 percent. An electric pump also offers the option to continue coolant circulation after a hot engine shutdown, potentially avoiding damage induced form excessive localized heating.

\section{DC-to-DC Converter}

The baseline configuration utilized a belt-driven alternator to provide power to the low voltage accessories. The alternator provided by the OEM exhibited an efficiency of approximately 46 percent. Thus, the alternator consumed twice the energy required to power the low voltage accessories. The TEPS truck utilized a DC-to-DC converter drawing from the high voltage bus to provide power for the low voltage accessories. The average overall conversion efficiency through the generator and the DC-to-DC converter was around 80 percent, yielding considerable savings versus the alternator.

Interestingly, the average power drawn during testing by the LV accessories was nearly identical for the baseline $(0.49 \mathrm{~kW})$ compared to the TEPS configuration $(0.5 \mathrm{~kW})$. The two configurations did, however, show a difference in current drawn to the LV circuit. Both tests employed a current sensor manufactured by LEM with an accuracy of 1 percent to measure current to the LV battery. The baseline current averaged 35 amps while the TEPS truck averaged 40 amps from the DC-to-DC converter during the test. This divergence reflects the difference in charging voltage as well as the accessories. The additional low voltage accessory loads on the TEPS truck included the supervisory controller, the transmission pump control electronics, the low temperature water pump, the cooling system drive control electronics, and the ISG drive control electronics. These components draw an average of $100 \mathrm{~W}$ altogether. The current measurements and the bus voltage measurements from the ECM were used to calculate the power and energy used during testing and, as before, the instantaneous engine efficiency was used to calculate the fuel consumption.

During baseline testing the OEM alternator was responsible for using 5 percent of the fuel consumed during the test. The DC-to-DC converter on the TEPS truck was responsible for consuming 2.4 percent of the fuel burned during the TEPS test. In this instance, using an efficient DC-to-DC converter and pulling power from the high voltage bus reduced the amount of fuel required to power the low voltage components by roughly 48 percent.

\section{Start/Stop}

One of the objectives of this project was to determine the potential benefit of shutting off the engine when it is not required. To obtain this type of operation and minimize the impact on what is presently considered normal operation, the flywheel mounted motor/generator was designed to provide sufficient torque to launch the vehicle. In addition, a power unit was designed to provide hydraulic power to the transmission to facilitate a launch assist type of operation. By shutting off the engine when it was not required, a reduction in 
fuel consumption of 4.8 percent was achieved over the baseline non-electrified truck during a test run.

Based on the measured fuel rate when idling, 0.59 $L$ of fuel would have been burned had the engine idled during the stops in the driving portion of the TEPS test. A savings of $0.59 \mathrm{~L}$ cannot be claimed, however, because substantial fuel was used restarting the engine and providing hydraulic power to the transmission during these stops. The amount of fuel necessary to restart the engine during the test totaled $0.44 \mathrm{~L}$ and about $0.04 \mathrm{~L}$ of fuel was required to maintain hydraulic power in the transmission. In total, around $0.48 \mathrm{~L}$ of fuel was used during the test to launch the vehicle, bringing total fuel savings from shutting down the engine during the driving portion of the test to 0.15 $L$ or roughly 0.8 percent of the fuel consumed. When one considers the battery losses that account for about $0.06 \mathrm{~L}$ of fuel, this value drops to 0.5 percent.

Further analysis showed that the motor was operating at an efficiency of around 25 percent at launch. Therefore, it would be reasonable to expect that by increasing the motor efficiency to 75 percent, a 1.5 percent reduction in fuel consumption would have been realized. Additional optimization of idle conditions for specific applications could also promote further savings.

\section{Source Losses}

The TEPS truck utilized multiple power sources as well as a power converter. The inefficiencies in these sources led to additional losses in the system. The generator had a theoretical efficiency around 93 percent in the operating range in which it was typically operating. The current sensor provided by the electronics in the energy storage system was used to obtain the current supplied by the generator. This current was used to determine the power output of the generator during the test. Based on the 93 percent generator efficiency, about 0.5 percent of the fuel consumed by the TEPS truck would have been converted to heat by the generator.

The high voltage energy storage system was also a source of electrical losses during the testing. The battery pack had an internal resistance typically around one half ohm which resulted in a loss proportional to the square of the current. During the test, the current to and from the battery was measured by the batteries internal electronics; this was used to determine the power. Based on measurements of the charge and discharge power of the battery, and the starting and ending pack state of charge, the pack was found to have an average efficiency of 92.6 percent over a charge and discharge cycle. As such, 0.3 percent of the fuel used during the TEPS test was converted to heat in the battery. Finally, the DC-to-DC converter, which was used to power the low voltage bus, exhibited an average efficiency of 86 percent, resulting in a loss of around 0.3 percent. The total of all the losses in the power sourcing devices sum to 1.1 percent of the fuel consumed during the course of the test.

The analysis of source losses does not account for brake energy reclamation. Operating the generator during braking events reclaims some energy to the TEPS truck. During testing at TRC, this energy proved sufficient to make up for the conversion losses in the generator, causing the generator to appear lossless. During higher speed operations, the contribution of brake energy might be even more significant on account of the additional kinetic energy available for recapture.

\section{Summary}

Throughout the course of the test, the savings due to the reduction of parasitic losses in the components summed to 13.65 percent of the fuel consumed by the TEPS truck. This 13.65 percent does not take into account the losses due to overhead, which are estimated at approximately 0.3 percent. The electrification of the fan provided the greatest reduction in fuel, at approximately 9.3 percent, followed by the DC-to-DC converter that saved 3.2 percent. The water pump provided a savings of 1.2 percent. The remaining fuel savings can be attributed to idle fuel reduction and brake energy reclamation. Idle fuel reduction, reduced fuel consumption during the driving portion by 3.0 percent and 5.6 percent during the extended idle portion of the test. An additional 0.5 percent was saved through reclaiming brake energy when compared to the non-electrified test truck. All told, the TEPS effort demonstrated a significant 15\% reduction in fuel consumption during testing at TRC.

\section{CONCLUSION}

The TEPS project demonstrated an integrated system of electric accessories and truck electrification to improve fuel economy. Electric accessories and systems have been implemented on a Class 6 medium-duty truck. The nucleus of the system is the ISG, which starts the engine and generates all on-board electric power. The machine presents a significant improvement in 
efficiency compared to the conventional alternator and offers an opportunity to provide additional power to the driveline.

ISG power generation to the 340 VDC bus enables the use of high voltage, high efficiency electric accessories. The accessories can operate in a more "on-demand" or variable fashion and offer more efficient operation in addition to packaging flexibility. Extensive supervisory control algorithms harness the capability of the ISG, energy storage, and electric accessories. Simulation results obtained by coupling a dynamic plant model with control algorithms suggested significant fuel consumption reductions for virtually any medium-duty application or driving cycle, particularly those with extended idle periods. Road testing confirmed these simulation findings with a measured 15 percent fuel efficiency improvement.

A 15 percent reduction in fuel consumption translates to 300 million gallons of fuel saved annually by the medium-duty trucks in the United States. These savings suggest an annual economic impact potential of $\$ 1.1$ billion to the medium duty truck market. Considering historical fuel pricing, the potential economic impact has nearly doubled since the TEPS project launched in October 2004. ${ }^{*}$ The TEPS team has demonstrated an integrated system of electric components that not only enhances functionality, but also provides substantial fuel savings without disrupting the vehicle traits expected by intermediate customers and end-users alike.

\footnotetext{
* Fuel pricing obtained from the U.S. Energy Information Administration. Truck volume data sourced from 2002 Vehicle Inventory and Use Survey.
} 


\section{APPENDIX A: REFERENCES}

1. Slezak, L., Program Manager, Truck Essential Power Systems Efficiency Improvements for Medium Duty Trucks, Department of Energy. Contract DE-FC26-04NT42258, Awarded August 2004.

2. Algrain, M.C., W.H. Lane, D.C. Orr. "A case study in the electrification of Class-8 trucks," Electric Machines and Drives Conference, 2003. IEMDC'03. IEEE International, Volume 2, 1-4 June 2003. Page(s): 647-655. Vol. 2.

3. Chalgren Jr., Robert D., David J. Allen. "Light Duty Diesel Advanced Thermal Management," 2005-01-2020, SAE International, Warrendale, PA, 2005.

4. Slone, L.M., Principal Investigator, Advanced Electric Systems and Aerodynamics for Efficiency Improvements in Heavy Duty Trucks, Department of Energy. Contract DE-FC26-04NT42189, Final Report January 2008. 


\section{APPENDIX B: PUBLIC RELEASES OF RESULTS}

"Electrification for Efficiency Improvements in Medium Duty Trucks" (06CV-117) presentation given by Larry M. Slone on 11/02/2006 at the Society for Automotive Engineers 2006 Commercial Vehicle Engineering Congress \& Exhibition. 
APPENDIX C: INVENTIONS/PATENT APPLICATIONS

\begin{tabular}{|c|c|c|c|}
\hline TITLE & INVENTOR & DATE REPORTED & $\begin{array}{l}\text { DOCKET NUMBER } \\
\text { APPLICATION } \\
\text { NUMBER }\end{array}$ \\
\hline Motor/Generator & Christopher D. Hickam & $5 / 11 / 05$ & S-108,047 \\
\hline $\begin{array}{l}\text { Automatic Transmission } \\
\text { Operation with Input } \\
\text { Shaft Mounted ISG }\end{array}$ & Christopher D. Hickam & $5 / 11 / 05$ & S-108,083 \\
\hline Shorting Connector & Christopher D. Hickam & $2 / 22 / 06$ & S-110,223 \\
\hline Bus Disconnect System & Christopher D. Hickam & $2 / 22 / 06$ & $\begin{array}{l}\text { S-110,224 } \\
\text { Caterpillar has decided } \\
\text { to not pursue this further }\end{array}$ \\
\hline $\begin{array}{l}\text { Electrical Interface } \\
\text { System }\end{array}$ & Christopher D. Hickam & $2 / 22 / 06$ & S-110,225 \\
\hline $\begin{array}{l}\text { Electrical Shorting } \\
\text { System }\end{array}$ & Christopher D. Hickam & $9 / 14 / 06$ & S-110,850 \\
\hline $\begin{array}{l}\text { Series Cooling Fan } \\
\text { Arrays in Engine } \\
\text { Cooling System }\end{array}$ & Christopher D. Hickam & $10 / 24 / 06$ & $\begin{array}{l}\text { S-110,993 } \\
\text { Caterpillar has decided } \\
\text { to not pursue this further }\end{array}$ \\
\hline
\end{tabular}

